TM-40

TOWARD A THEORY OF GEOMETRIC TOLERANCING

\author{
by \\ Aristides A. G. Requicha \\ PRODUCTION AUTOMATION PROJECT \\ March 1983 \\ Reprinted August 1988 \\ Reprinted December 1988
}



Technical Memorandum No. 40

\author{
PRODUCTION AUTOMATION PROJECT \\ College of Engineering \& Applied Science \\ The University of Rochester \\ Rochester, New York 14627
}

\title{
TOWARD A THEORY OF GEOMETRIC TOLERANCING
}

by

Aristides A. G. Requicha

March 1983

The work described in this document was supported primarily by the National Science Foundation under Grant ECS-8104646 and by companies in the Production Automation Project's Industrial Associates Program. Any opinions, findings, conclusions or recommendations expressed in this publication are those of the author and do not necessarily reflect the views of the National Science Foundation or the Industrial Associates of the P.A.P. 



\begin{abstract}
Manual drafting is rapidly being replaced by modern, computerized systems for defining the geometry of mechanical parts and assemblies, and a new generation of powerful systems, called Geometric (Solid) Modelling Systems (GMISs), is entering industrial use. Solid models are beginning to play an important role in off-line robot programming, model-driven vision, and other industrial robotic applications.

A major deficiency of current GMSs is their lack of facilities for specifying tolerancing information, which is essential for design analysis, process planning, assembly planning for tightly-toleranced components, and other applications of solid modelling. This paper proposes a mathematical theory of tolerancing that formalizes and generalizes current practices, and is a suitable basis for incorporating tolerances in to GMSs.

A tolerance specification in the proposed theory is a collection of geometric constraints on an object's surface features, which are two-dimensional subsets of the objects' boundary. An object is in tolerance if its surface features lie within tolerance zones, which are regions of space constructed by offsetting (expanding or shrinking) the object's nominal boundaries.
\end{abstract}





\section{TABLE OF CONTENTS}

1. Introduction . . . . . . . . . . . . . . . 1

2. Current Practices and Prior Work . . . . . . . . . . . . . . . 2

3. A Theory of Tolerancing . . . . . . . . . . . . . . . . . . . 4

3.1 Outline . . . . . . . . . . . . . . . . 4

3.2 Variational Classes and Tolerance Specifications . . . . . . . . .6

3.3 Extended and Symmetric Features . . . . . . . . . . . . . 8

3.4 Datum Systems . . . . . . . . . . . . . . . . . . . 15

3.5 Offset Solids, Size Tolerances, MMC and LMC . . . . . . . . 16

3.6 Form, Orientation, and Runout Tolerances . . . . . . . . . . 20

3.7 Position Tolerances . . . . . . . . . . . . . . . . . . 23

3.8 Validity of Tolerance Specifications . . . . . . . . . . . . 25

4. Conclusions . . . . . . . . . . . . . . . . . . . . . . . 26

References . . . . . . . . . . . . . . . . . . . . . . . . . 28 



\section{Introduction}

The traditional medium for specifying mechanical parts and assemblies are blueprints (engineering drawings), which contain graphical descriptions of the nominal or ideal parts plus tolerancing information that defines allowable departures from the nominal objects. Tolerances are specified by designers, and should ensure that parts "in spec" are functionally equivalent and interchangeable in assembly. Tolerancing information is essential for planning part manufacture and "tight" assembly operations, for part inspection, and for other design and production activities.

Manual drafting is rapidly being replaced by modern, computerized systems for defining the geometry of mechanical parts and assemblies, and a new generation of powerful systems, called Geometric (Solid) Modelling Systems (GMSs), is entering industrial use [REQU82]. Solid models generated by Computer Aided Design (CAD) are expected to become the primary source of geometric information in integrated design and production systems, and solid modelling technology is beginning to play an important role in off-line robot programming, model-driven vision, and other industrial robotic applications.

Current GMSs lack tolerancing facilities, and therefore can neither support fully automatic manufacturing planning nor some of the spatial reasoning required for assembly planning. For example, analysis of manipulator and sensor inaccuracies must be supplemented with analysis of part inaccuracies for tightfitting assembly tasks [BROO82]. To incorporate tolerancing information in GMSs and use it in automatic analysis and planning, the semantics of tolerances should be defined mathematically. General definitions, which can be "understood" by general programs, are preferable to special-case definitions, which are difficult to imbed in programs and lead to large amounts of possibly inconsistent code. Unfortunately, current industrial tolerancing practices, described in standards [ANSI73] and texts [LEVY74], are defined informally, mainly for special situations.

The goal of this paper is to propose a theory of tolerancing that formalizes and generalizes current practices, and is a suitable basis for incorporating tolerances into GMSs. The theory was designed to follow established tolerancing practices as closely as possible, but some departures seemed desirable and others unavoidable. The paper also explores briefly the representational implications of the theory, which are straightforward, but does not attempt to discuss its algorithmic implications, which are presently unknown.

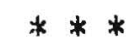




\section{Current Practices and Prior Work}

Current industrial tolerancing practices use a mix of so-called "geometric tolerances", sometimes called "modern" or "true-position" tolerances, with "conventional" $(+/-)$ tolerances - see Figure 2-1 for a very simple example. The current trend in industrial practice is toward an increased use of geometric tolerancing.

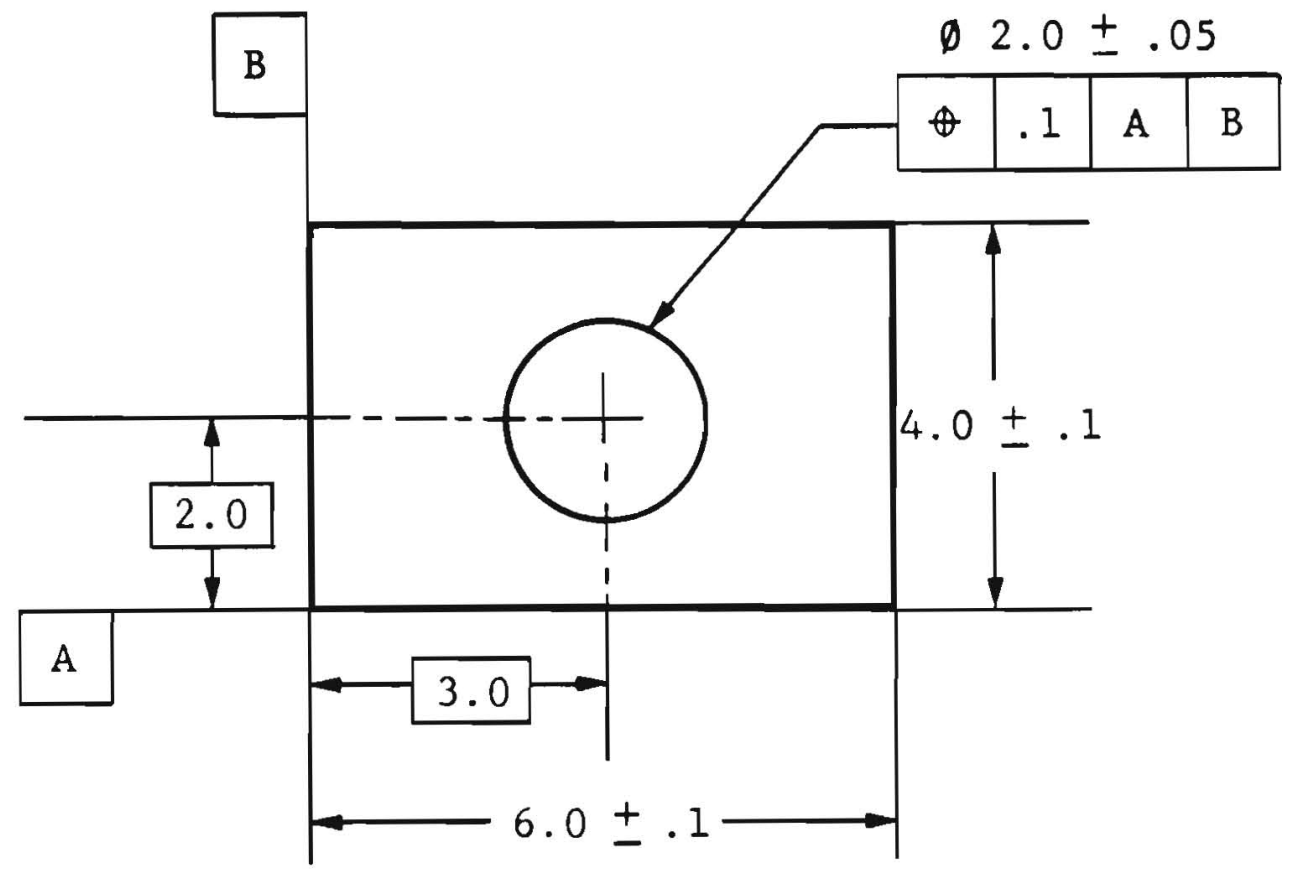

Figure 2-1

A simple example of current tolerancing practices.

The rectangle and circle dimensions are specified by conventional $+/$ - tolerances. The symbol block associated with the circle specifies a "true-position" tolerance with respect to datums $A$ and $B$, which are the surfaces labelled in the drawing.

Much of modern geometric tolerancing is easy to formalize, but there are significant gaps centered on the notions of "feature" and "size". Conventional tolerancing, however, appears to be inherently ambiguous. Experienced humans usually can resolve tolerancing ambiguities by appealing to "implicit datums" and implied geometric relationships (e.g. tangency), but it is unreasonable to expect automata to make similar decisions.

Some of the issues that are inadequately addressed in current practices but 
should be resolved in a theory of tolerancing are the following.

- What is a feature? Features are the entities to which tolerances apply, yet standards and texts on tolerancing provide no precise definition of "feature".

- What is the spatial extent of "tolerance zones"? Texts suggest, for example, that to test a vertical hole one should (conceptually) use a gage with height equal to the nominal hole height; however, this may result in portions of an object's boundary being untested.

- How does one associate datums to features more complex than simple holes or planes?

- What is the "size" of an actual physical feature? For example, what is the diameter of an actual (not perfectly cylindrical) hole? Can "size" be defined for complex features? How?

- What do $+/$ - tolerances mean when features have imperfect form?

- How are MMC (Maximum Material Condition) and LMC (Least Material Condition) defined for complex features?

Published studies of tolerancing in the contest of geometric modelling are very few. My own report [REQU77a] addressed both conventional and geometric tolerancing but provided no clues on how to deal simultaneously with both. Hillyard's Ph.D. dissertation. [HILL78a], [HILL78b], [HILL78c], and Hoffman's work [HOFF82] appear to be concerned exclusively with conventional tolerances and do not address issues such as those listed above. Work on geometric constraints and object parameterization (see e.g. [BROO81], [FITZ81] and [LIN81]) also appears to be relevant to tolerancing. The approach that I shall discuss below evolved from my earlier research [REQU77a] and is not related to any of the other published work cited above. (A forthcoming report [REQU83] will discuss mathematical and computational issues raised by tolerancing and alternative theoretical approaches.) 


\section{A Theory of Tolerancing}

The following subsections propose a mathematical theory of tolerancing suitable for computational implementation. Two of the main (and perhaps surprising) tenets of the theory are the following:

- Conventional tolerances are subsumed into geometric tolerances that contain no "implicit datums" or unstated constraints. ${ }^{3-1}$

- There is no formal notion of a "measured size", although "size tolerances", $\mathrm{MMC}$ and similar notions are defined mathematically.

First I shall present a rough outline of the theory, and then embark on a detailed exposition.

\subsection{Outline}

Consider initially a solid cylinder in 3-D Euclidean space. The size of the cylinder obviously is well defined and can be described by its radius and height. We can also agree on an unambiguous way, and a corresponding set of parameters (distances and angles), to define the cylinder's position, i.e., its location and orientation.

Consider now a family of (perfect-form) cylinders with sizes and positions close to those of the initial cylinder. It is clear that such a family can be defined in terms of a range of allowable values for the agreed size and positional parameters of cylinders. This seems to be the spirit of conventional tolerancing practices and also of the geometric modelling literature cited above. Specifically, a manufactured solid is "in spec" if its defining parameters are within specified ranges.

Manufacturing processes, however, do not produce perfect cylinders. Therefore, let us consider an object that results from a small but random deformation of our initial cylinder. It should be clear that the notions of "size" and "position" for such an object have no obvious meanings. A possible way out of this impasse consists of defining the size and position parameters of an imperfectform object as the corresponding parameters of an associated perfect-form object, generated by surface-fitting or similar techniques. This approach is sometimes useful, e.g. for defining "datums" (see Section 3.4 below), but does not seem applicable to most of the modern tolerancing practices.

3-1 This does not mean that human users of GMSs must specify explicitly all constraints - good user interfaces can take care of many of these through defaults. 
The approach I shall develop below does not attempt to assign well-defined sizes or positions to imperfect-form objects. It considers an imperfect-form object "in spec" when its boundary is within "tolerance zones", which are defined in terms of perfect-form objects. To flesh out the approach, one needs general mathematical procedures for building tolerance zones around objects and for specifying how such zones are located and oriented.

As a preface to the detailed discussion in the following subsections consider a simple cylinder of radius $R$, various tolerance specifications on it, and the associated tolerance zones.

1) Size tolerance $T_{s}$. A surface (generally not a perfect cylinder) satisfies this specification if it lies entirely within an annular tolerance zone defined by two coaxial cylinders of radii $R+T_{s} / 2$ and $R-T_{s} / 2$. The location and orientation of the tolerance zone are arbitrary, i.e., can be adjusted to ensure (hopefully) that the surface fits in the zone.

2) Form tolerance $T_{f}$. The tolerance zone is an annulus defined by two coasial cylinders of radii $R_{1}$ and $R_{2}$, with $R_{1}-R_{2}=T_{f}$, and positioned arbitrarily. $R_{1}$ and $R_{2}$ are unrelated to $R$. Observe that a surface that satisfies a size tolerance $T_{s}$ also satisfies a form tolerance $T_{f}=T_{\sigma}$, but the converse is not generally true.

3) Orientation tolerance $T_{o}$ with respect to a coordinate system. The tolerance zone is similar to a form tolerance zone, but its orientation in the specified coordinate system is fixed. (The location is arbitrary.) An orientation tolerance implies a form tolerance, but not conversely.

4) Position tolerance $T_{p}$ with respect to a coordinate system. The tolerance zone is the annulus defined by two coaxial cylinders of radii $R+T_{p} / 2$ and $R-T_{p} / 2$, correctly located and oriented with respect to the given coordinate system. A position tolerance implies size, form and orientation tolerances, but the converses generally are not true. (There are less restrictive position tclerances - see Section 3.7.)

Observe that the tolerance zones in all of the examples above were obtained by "offsetting" the perfect-form surface. ${ }^{3-2}$

3-2 Offsetting seems to be a very useful concept in geometric modelling. It has found applications in cutter-path generation for machine tools, mass-property calculation [LEE82], and trajectory planning for robots [LOZA79]. 
The particular offsetting technique used in the proposed theory is described in Section 3.5. It is general, very simple, and always produces well-defined tolerance zones. However, much of the theory is independent of the specific offsetting procedures used. above.

The following subsections elaborate and formalize the rough outline presented $* *$

\subsection{Variational Classes and Tolerance Specifleations}

The goal of a tolerance specification is to define a class of objects that are interchangeable in assembly operations and functionally equivalent. Such classes of objects will be called in this paper variational classes [REQU77a]. A tolerance specification is an entity of a computational nature: it is a representation of a variational class. (See [REQU80] for basic representational concepts.) I propose in this paper tolerance specifications that consist of:

1) An unambiguous representation for a nominal solid $S$ (an $r$-set [REQU77b] [REQU80]).

2) A representation for a decomposition of $\partial S$ (the boundary of $S$ ) into subsets $F_{i}$ called nominal surface features, that are homogeneously 2-D [REQU77b] and whose union is $2 S$.

3) A collection of geometric assertions $A_{i j}$ about $S$ 's nominal surface features.

The specific schemes used to represent solids and their surface features are unimportant for the purposes of the theory, but solids are assumed to be definable through regularized Boolean operations and rigid motions on a finite set of prinitive half spaces, whose boundaries are called primitive surfaces [REQU77b, REQU80]. Surface features and assertions must possess certain properties discussed later in this paper. (Some 2-D subsets of $\partial S$ cannot be features.)

The semantics (geometric meaning) of a tolerance specification will be defined by a mathematical rule - called a theoretical inspection procedire - for answering the following question: Given a tolerance specification $T=$ $\left(S,\left\{F_{i}\right\},\left\{A_{i j}\right\}\right)$ and a subset $P$ of $E^{3}$ that models an actual (physical) manufactured part, is $P$ in the variational class defined by the specification $T$ ?

A (model of a) part $P$ satisfies a tolerance specification $T$ if and only if there is a decomposition of $\partial P$ into subsets $G_{i}$ called actual surface features, such that: 
1) $\cup G_{i}=\partial P$.

2) There is a one-to-one correspondence between $G_{i}$ and the nominal surface features $F_{i}$ of $S$.

3) Each $G_{i}$ satisfies the assertions $A_{i j}$ associated with its corresponding $F_{i}$.

Observe that this definition ensures that the entire boundary of a part $P$ is taken into consideration, but does not prescribe a unique segmentation of $P$ 's boundary into actual features.

A nominal feature $F$ is simple if it lies in a single primitive surface. A feature is composite if it is the union of simple features. Note that the simple features that contribute to a composite feature need not lie in distinct primitive surfaces (although they usually do). Actual features that correspond to simple nominal features also are called simple, and similarly for composite features.

I shall assume that the bounding surfaces of manufactured objects satisfy a "slow variation" constraint that ensures that a surface does not vary too rapidly (at a scale comparable to the applicable tolerance values), and therefore does not create "nicks" or thin "slivers" as shown in Figure 3-1. (I have not tried to formalize this constraint; a formal definition should exclude "slivers" but consider low-amplitude high-frequency variations acceptable.) 


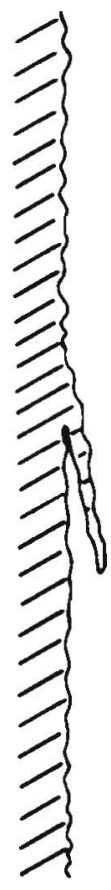

(a)

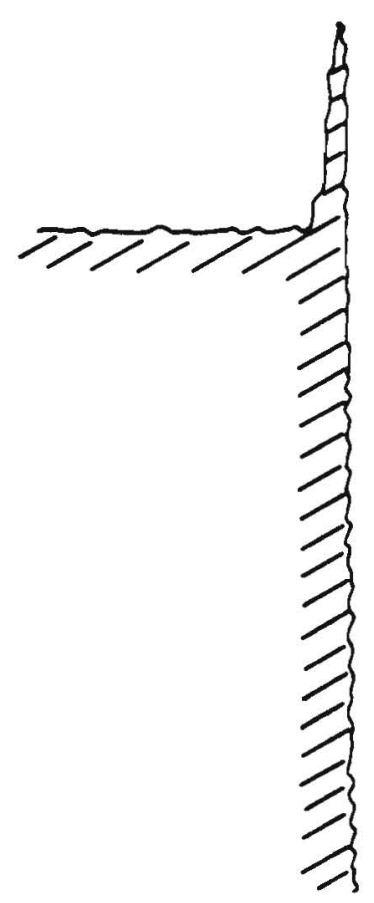

(b)

Figure 9-1

Violations of the slow variation assumption

in a "face" (a) and at an "edge" (b)

\subsection{Extended and Symmetric Features}

Assertions about features often establish constraints on the geometric relationships between bounded portions of a physical object's boundary and unbounded surfaces, centerplanes, axes, and similar geometric entities. These notions are formalized in this section.

First I introduce the notion of extended feature by means of an example and then formalize it. Consider the nominal surface feature that consists of the two faces of the slot shown in Firgure 3-2a. The extended feature corresponding to $F$ consists of the two unbounded planes shown in projection in Figure 3-2b, together with information on "where the material is". More precisely, the extended leature is an unbounded solid defined as the union of the planar halfspaces shown in Figure 3-2b. 


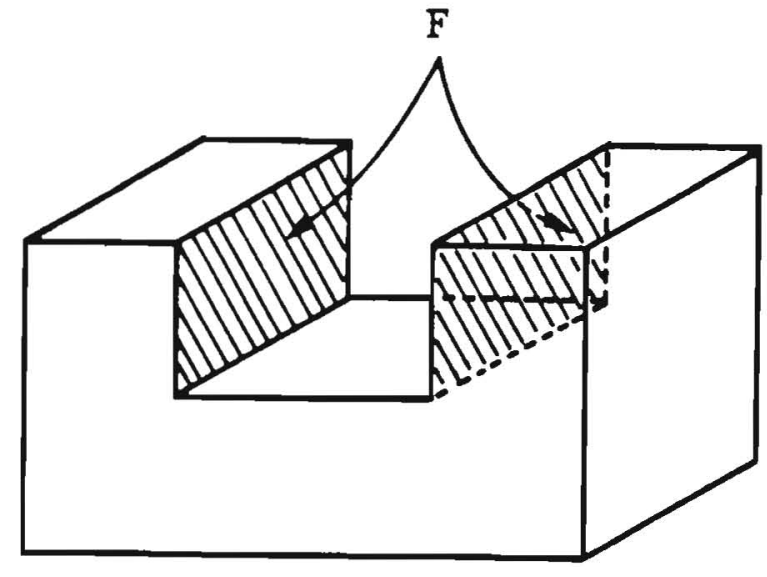

(a)

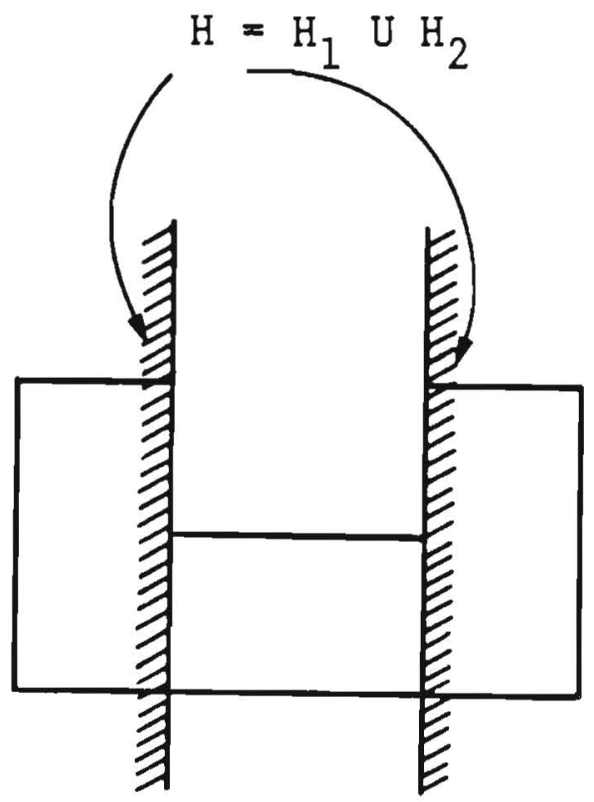

(b)

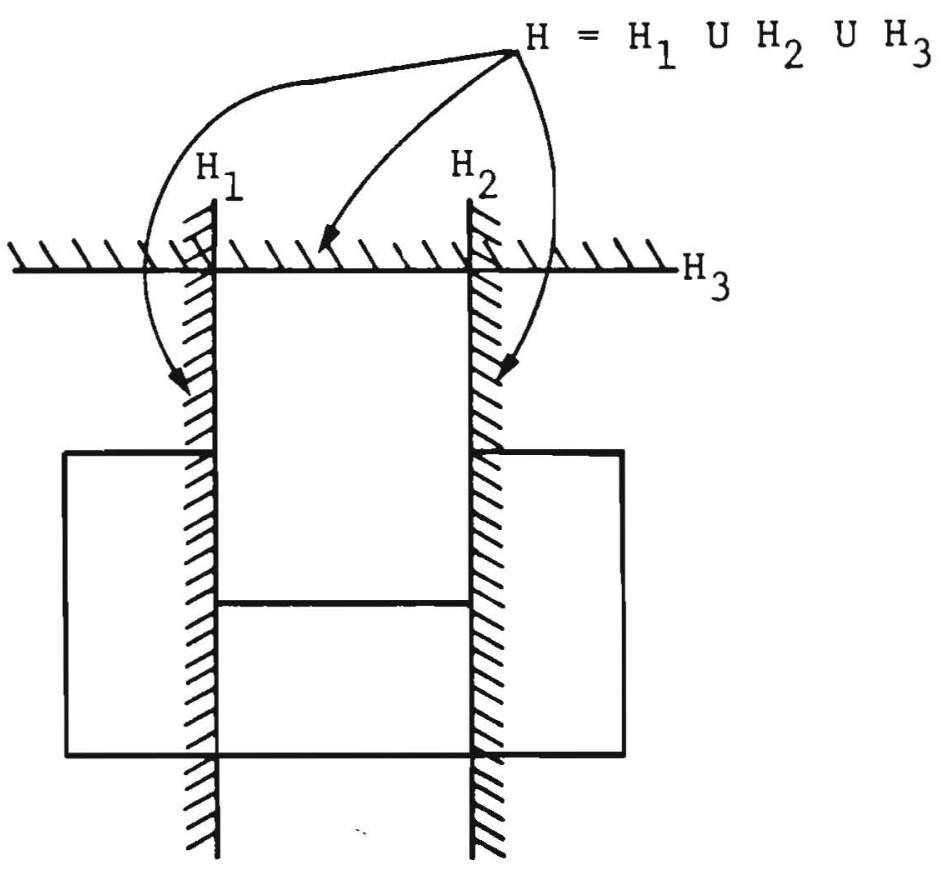

(c)

Figure 9-2

A slot (a), its associated extended feature (b), and a set that violates the defining conditions for extended features (c) 
A general definition follows. An extended feature $H$ associated with a nominal surface feature $F$ is a solid (possibly unbounded) defined as a Boolean composition of halfspaces $H_{i}$ and satisfying the following conditions:

1) $\partial H \supset F$.

2) $H$ must not contain in its definition halfspaces $H_{i}$ that contribute 2-D subsets to $\partial H$ but not to $F$.

3) Define the "neighborhood of $p$ with respect to $S$ " as $N(p, S ; R)=$ $B(p ; R) \cap S$, where $B(p ; R)$ is a ball of radius $R$ centered on $p$, and define similarly a neighborhood with respect to $H$ [TILO80]. Then, for every point $p$ in the (2-D) interior of the feature $F$, and for $R$ sumiciently small, $N(p, S ; R)=N(p, H ; R)$. (This condition says essentially that the "material sides" of $S$ and $H$ must agree.)

In the example above the solid $H$ shown in Figure 3-2c violates condition 2 because $H_{3}$ contributes a "face" to $\partial H$ but not to $F$. Note, however, that an extended feature may contain halfspaces that contribute neither to $\partial H$ nor to $F$. The halfspace $\mathrm{H}_{2}$ of Figure 3-3 provides an example.

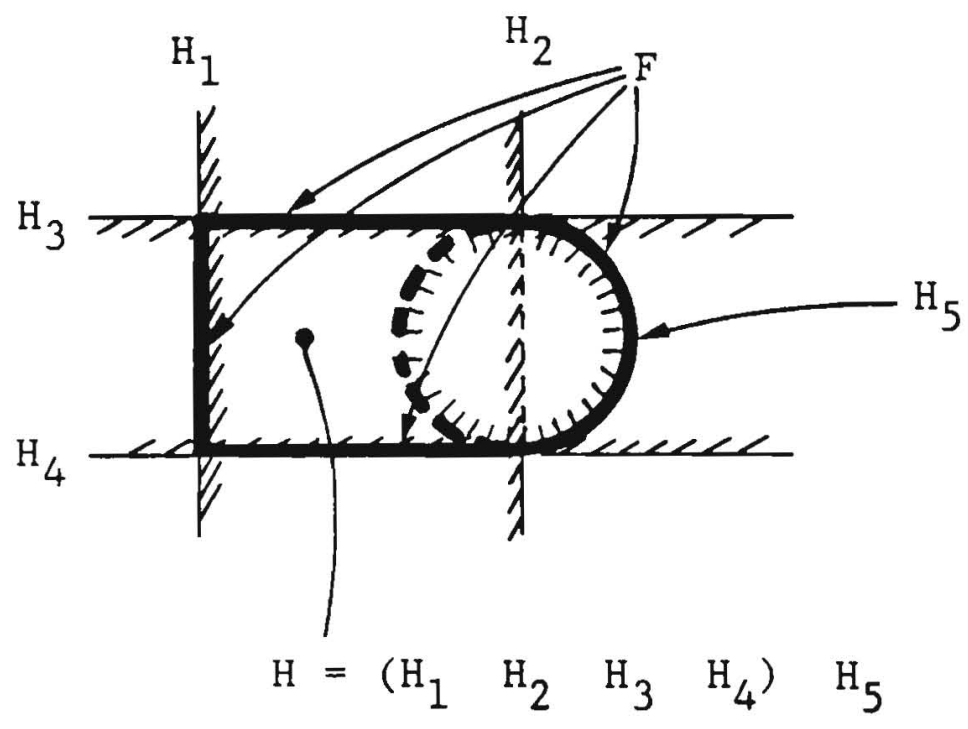

Figure $9-9$

The planar halfspace $H_{2}$ contributes neither to $F$ nor to the boundary of $H$, yet it cannot b omitted in the Boolean composition that defines 
It can be shown that, under mild assumptions, the halfspaces that do contribute 2-D subsets to $\partial H$ - called b-components [REQU77a] - are uniquely defined (up to complementation) by the feature $F$ [SILV82]. Halfspaces that are not $b$-components are not unique but this causes no problems.

Observe that certain 2-D subsets of a solid's boundary cannot be associated with an extended feature. The union of the cylindrical surfaces of the boss and hole shown in Figure 3-4 provides an example. Nominal surface features are required to be homogeneously $2-D$ and to have associated extended features, and $F$ in Figure 3-4 therefore is not an acceptable feature.
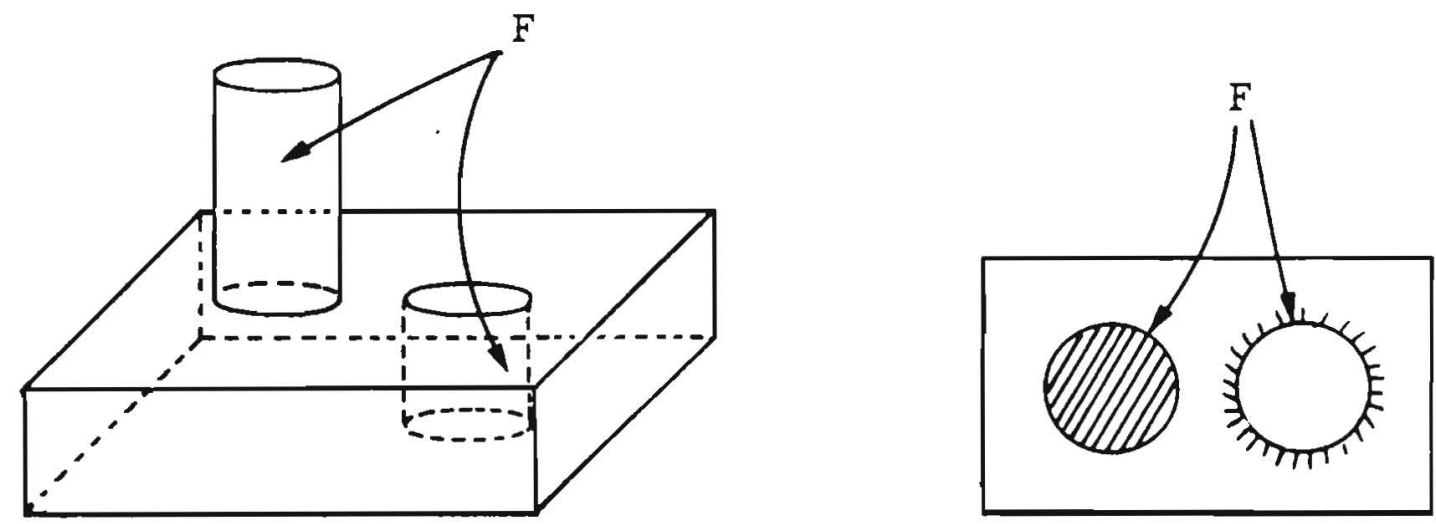

Figure $9-4$

Two cylindrical surfaces that have no corresponding extended feature and therefore are not a nominal surface feature.

Extended features that exhibit symmetry are especially interesting because they can be used to establish "datums", as explained below, and because they may have identifiable "centerplanes", "centerlines", or "centers" which can be constrained by assertions.

For simplicity, the definitions below apply to symmetry about the principal planes, axes and origin of a master coordinate system, but generalizations are obvious. An extended feature $H$ is symmetric with respect to the $x y$ plane if it is invariant under the reflection $z \rightarrow-z$; the plane $x y$ is then called a centerplane for $H$ (and also for its associated nominal surface feature $F$ ). $H$ is symmetric with respect to the $z$ axis if it is invariant under the transformation $x \rightarrow-x, y \rightarrow$ $-y$; the $z$ axis is the feature's centerine or axis. Finally, $H$ is symmetric with respect to the origin $O$ - called the feature's center - if it is invariant under the transformation $x \rightarrow-x, y \rightarrow-y, z \rightarrow-z$. 
Figure 3-5 shows centerplanes and centerlines for two features. Observe that the features shown in the figure also have an infinite number of centerplanes parallel to the paper.

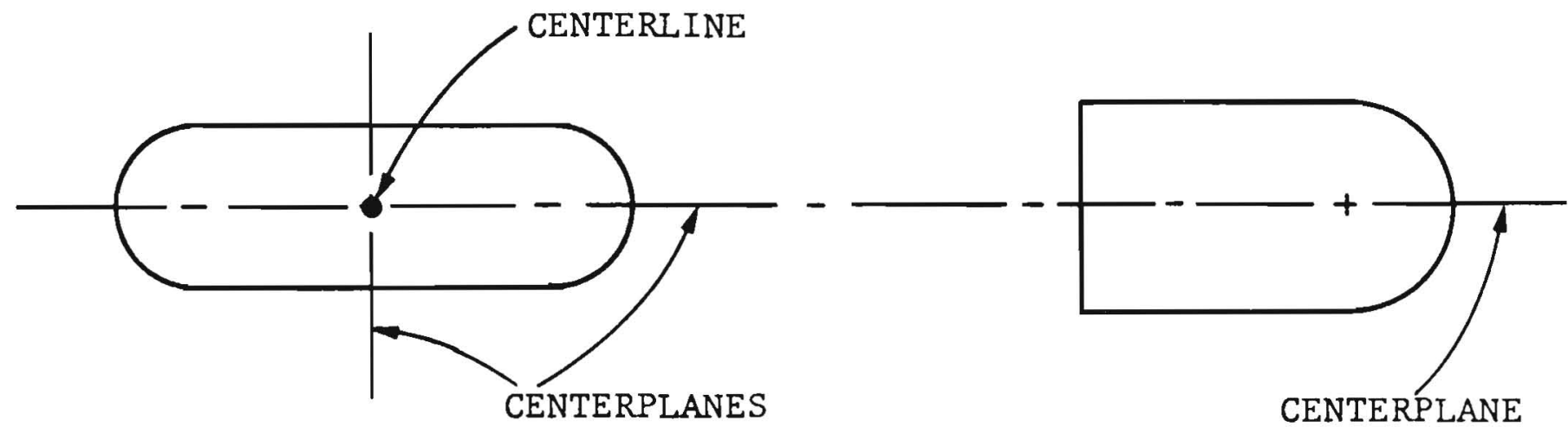

(a)

(b)

Figure 9-5

Two symmetric features (shown in 2-D projection).

Experienced humans can infer from blueprints which azes or centerplanes are relevant to a tolerance specification, but in computerized systems it may be more reasionable to require that the axis or centerplanes be specified explicitly, through statements similar to: "The centerplane of feature $F$ that passes through point; $O$ and is normal to vector $V$ ".

For symmetric features one can define the concepts of measured axes (or centerplanes, or centers) by formalizing the current practice of using expanding mandrels to determine hole centers. Specifically, let us consider an actual subset $G$ of a part's boundary and its corresponding surface feature $F$, extended feature $H$ and its centerplane $C_{p}$, which we assume to be the $x y$ plane. The measured centerplane of $G$ corresponding to $C_{p}$ is defined as follows. Consider a family of solids $H_{k}$ such that each $H_{k}$ is obtained from $H$ by a 1-D scaling transformation $x \rightarrow x, y \rightarrow y, z \rightarrow k z .^{3-3}$ We seek a solid $H_{k}$ with minimal $k$ and such that a congruent version $H_{k}^{\prime}$ of $H_{k}$ encloses completely $G$. The measured centerplane of $G$ is then the appropriate centerplane of $H_{k}^{\prime}$. Figure 3-6 illustrates the concept for a slot. Observe that the location and orientation of ${ }^{\prime} I_{k}^{\prime}$ are selected to minimize $k$.

3-3 Scaling transformations are the only reasonable formalization I have been able to find for capturing the notion of an "expanding feature". Because the effects of scaling depend on the choice of origin or axes I required features to be symmetric so as to have "natural" origins and axes. A general theory for non-symmetric features could be constructed by specifying explicit origins and axes. 


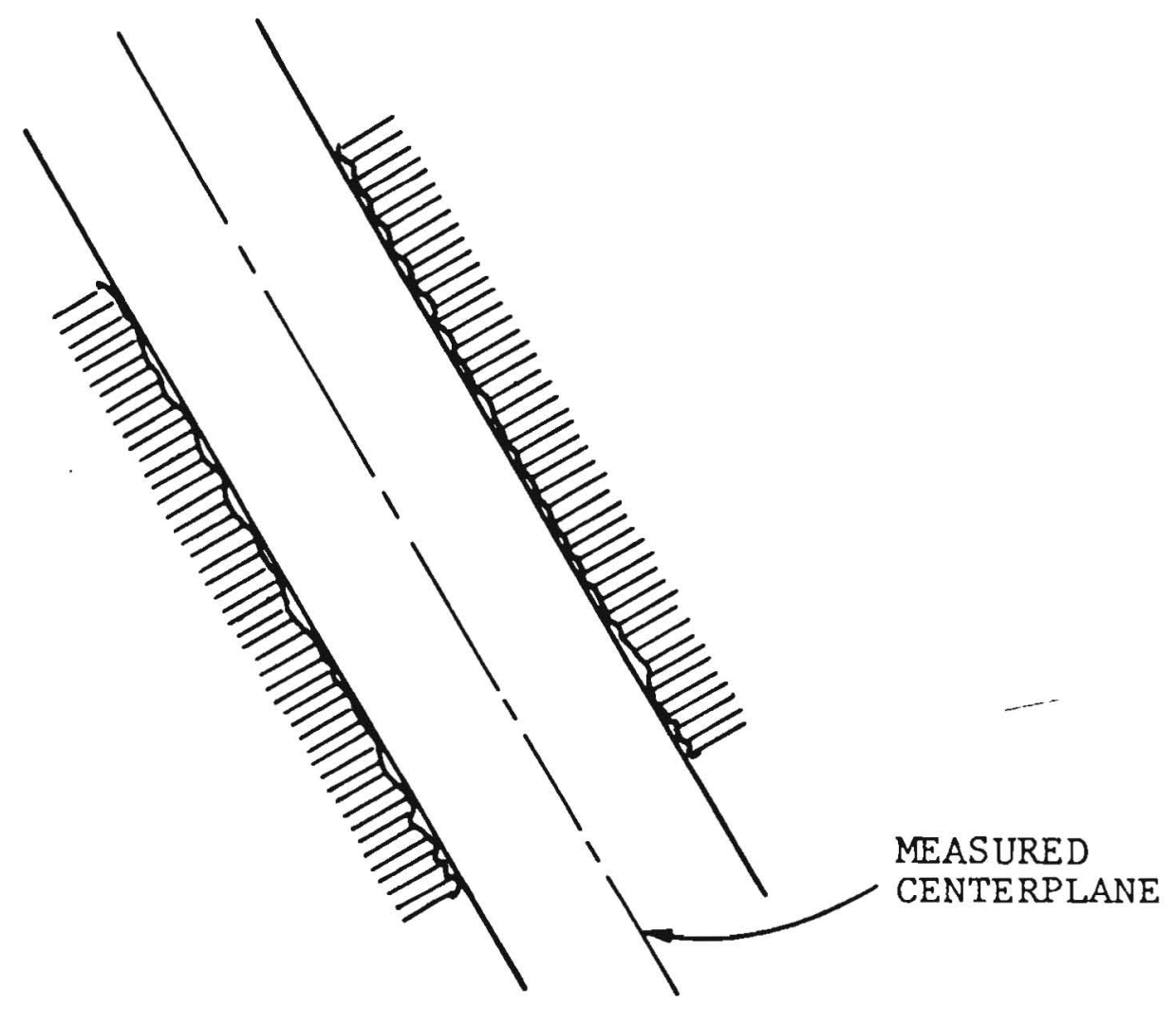

Figure 9-6

The measured centerplane for an actual slot feature.

Centerlines are defined similarly, by using 2-D scalings of the form $x \rightarrow$ $k x, y \rightarrow k y, z \rightarrow z$, where the axis is assumed to be the $z$-axis. For centers we use 3 -D scalings $x \rightarrow k x, y \rightarrow k y, z \rightarrow k z$, where the center is assumed to be the origin.

Readers should convince themselves that the above definitions capture mathematically current procedures for such simple features as holes and slots, and apply to any symmetric feature, no matter how complez.

Features that lie in a single planar surface (henceforth called simply "planar features") are not symmetric in the sense defined above. For such features I introduce the notion of a measured plane, which is analogous to a measured centerplane for a symmetric feature. Specifically, the measured plane associated with an actual planar feature $G$ is $\partial H$ where $H$ is a planar halfspace that encloses 
$G$ completely and whose boundary is "closest" to $G$ in some appropriate sense. In current practice $\partial H$ is selected so as to "just rest" on or "be tangent" to $G$, and sometimes points or lines where the actual feature should contact an ideal plane are specified explicitly. A more precise requirement might be that the (integral) sum of the distances of all points of $G$ to $\partial H$ be minimal. Figure 3-7 shows two examples.

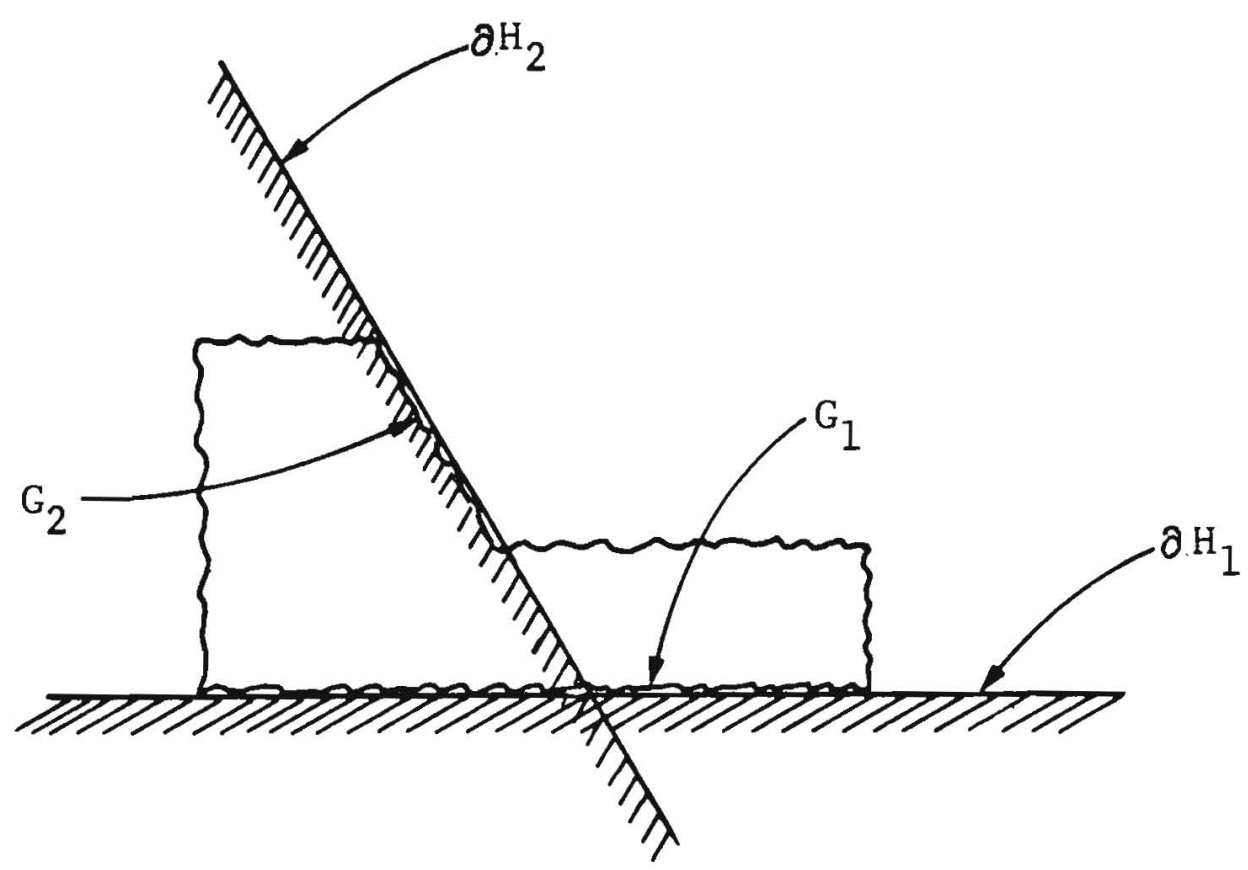

Figure 9-7

Two planar features $G_{1}$ and $G_{2}$, and associated measured planes $\partial H_{1}$ and $\partial H_{2}$.

Finally, it should be noted that measured entities (planes, centerlines, and so on) sometimes are not uniquely defined. When there is ambiguity a tolerance specification will always be interpreted to mean that there is (at least one) measured entily for which the appropriate assertions are satisfied. With this understanding, Ior simplicity of language I shall refer to "the measured entity" in the sequel as if it were unique. 


\subsection{Datum Systems}

The notions of datum and datum systems are well described in current standards and texts [ANSI73]. In the context of this paper a datum is a measured azis, centerplane, or center associated with a symmetric feature, or a measured plane associated with a planar feature.

The location and orientation (attitude) of features is controlled by assertions involving the geometric relationships of a feature to a datum or system of datums. The semantics of such assertions is straightforward. Suppose, for example, that a datum $D_{1}$ is associated with a feature $F_{1}$, and another feature $F_{2}$ is related to $D_{1}$ via an assertion. Given actual features $G_{1}$ and $G_{2}$ corresponding to $F_{1}$ and $F_{2}$, one constructs from $G_{1}$ the measured centerplane (or axis, ...) as explained in Section 3.2 and uses it as a datum to check whether $G_{2}$ satifies the assertion.

Ordered datum systems can be accommodated by modifying slightly the "measuring procedure" of Section 3.2. Consider the example of Figure 3-8. The measured plane corresponding to $G_{1}$ - the primary datum - is constructed as in Section 3.2, and the secondary datum corresponding to $G_{2}$ is constructed by a similar procedure but is constrained to be normal to the first.

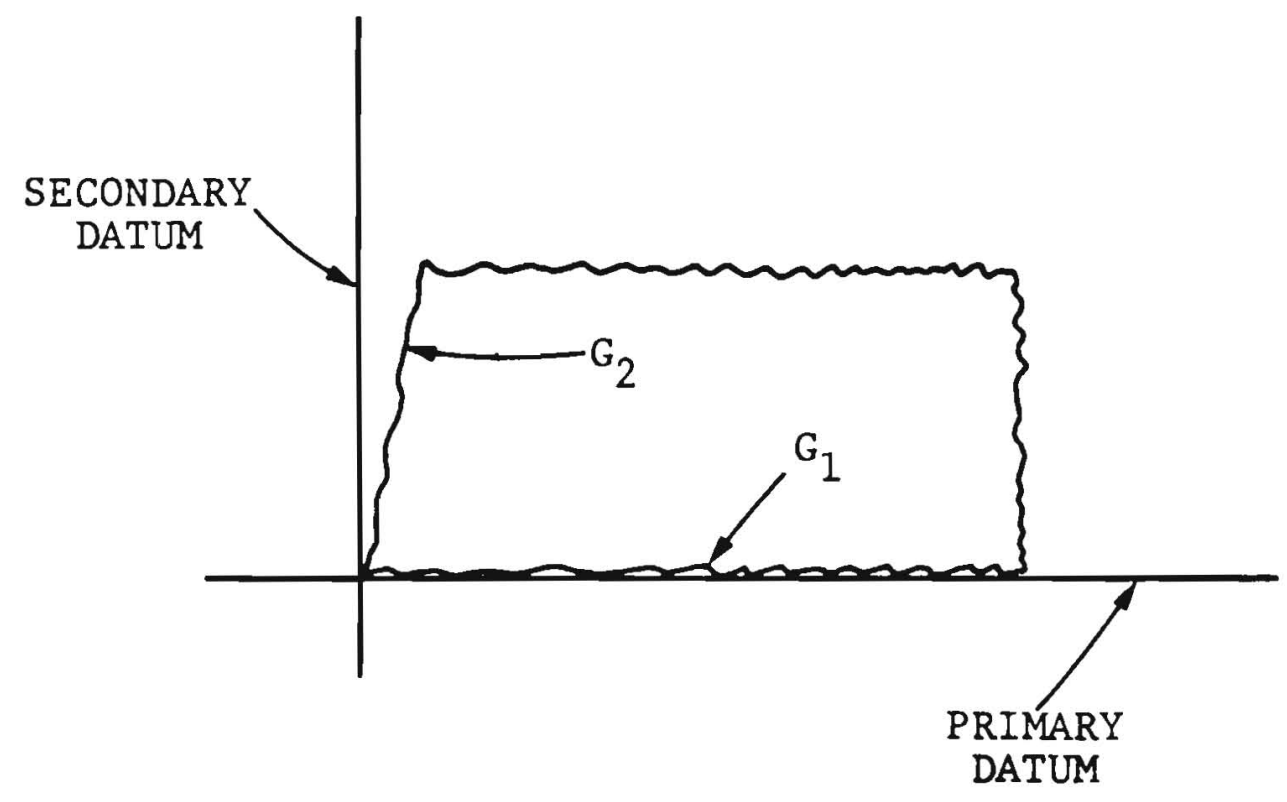

Figure 9-8

An ordered datum system.

The discussion above pertains to so-called "RFS datums". (RFS stands for 
"regardless of feature size" [ANSI73].) "Floating datums" (MMC) are also useful and can be accommodated essentially as explained in the standards [ANSI73].

The proposed theory departs from current practice in that it requires that each part have an explicitly defined "master" 3-plane datum system, and that all features be located from the master datum system or from explicitly specified datum systems related to the master. For example, one can locate a feature with respect to the master datum system, use this feature to construct a new datum, use the new datum to locate another feature, and so on. Therefore, what I propose is something akin to a tree of datum systems, rooted at the master datum system. (The datum graph is not really a tree because there are datums that are not part of complete datum systems, and so forth.)

\subsection{Offset Solids, Size Tolerances, MMC and LMC}

This section contains the major departures of the proposed theory from current practices. In essence, I reject the notion of a "measured size" and provide instead mathematical rules for deciding whether a feature satisfies a "size tolerance specification". The rules are based on notions of Maximum Material Condition (MMC) and Least Material Condition (LMC).

A few definitions are needed. First recall that the distance of a point $p$ of $E^{3}$ to a subset $S$ of $E^{3}$ is [NADL78]

$$
\operatorname{Dist}(p, S)=\min _{q \in S} \operatorname{Dist}(p, q),
$$

where $\operatorname{Dist}(p, q)$ is the ordinary point distance in $E^{3} \cdot 3^{3-4}$ Observe that the definition above implies that all points of $S$ are at zero distance from $S$, and that when $S$ is a solid ( $r$-set) the distance between an external point and the solid is the same as the distance between the point and the boundary of the solid, i.e.,

$$
\operatorname{Dist}(p, S)>0 \Rightarrow \operatorname{Dist}(p, S)=\operatorname{Dist}(p, \partial S) \text {. }
$$

Now let $D$ be a positive number and $S$ a solid, and define the corresponding (positive) single-offset solid $O(D ; S)$ as

$$
O(D ; S)=\{p: \operatorname{Dist}(p, S) \leq D\} \cdot \cdot^{3-5}
$$

3-4 If $S$ is not sufficiently smooth one must replace the minimum in the above definition by the infimum, or greatest lower bound.

3-5 Positive single-offset solids are sometimes called "generalized balls" in the mathematical literature [NADL78] and are closely related to Minkowski sums, which are used for example in geometric probability theory. 
For a negative offset $D$ a (negative) single-offset solid is defined as

$$
O(D ; S)=S-{ }^{*} O\left(|D| ; c^{*} S\right) \text {, }
$$

where - ${ }^{*}$ and $c^{*}$ denote regularized difference and complement. (Regularized set operators are slightly modified versions of their usual counterparts [REQU77b, REQU80].) Given a nominal feature $F$, its associated extended feature $H$, and two numbers $D_{p}>0$ and $D_{n}<0$ called the "positive and negative offsets", the MMC and LMC solids are defined as the appropriate offset solids, viz.,

$$
\begin{aligned}
& \operatorname{MMC}\left(D_{p} ; H\right)=O\left(D_{p} ; H\right), \\
& \operatorname{LMC}\left(D_{n} ; H\right)=O\left(D_{n} ; H\right) .
\end{aligned}
$$

Figure 3-9 provides a simple example. For a cylindrical hole feature of nominal radius 8 , positive offset .1 and negative offset -.1 , the corresponding MIMC and LMC solids are unbounded cylindrical halfspaces of radius 7.9 and 8.1, respectively. Intuitively, the MMC solid is the result of adding to the extended feature $H$ a layer of material of thickness $D_{p}$ and the LMC solid is the result of subtracting from $H$ a layer of thickness $\left|D_{n}\right|$.

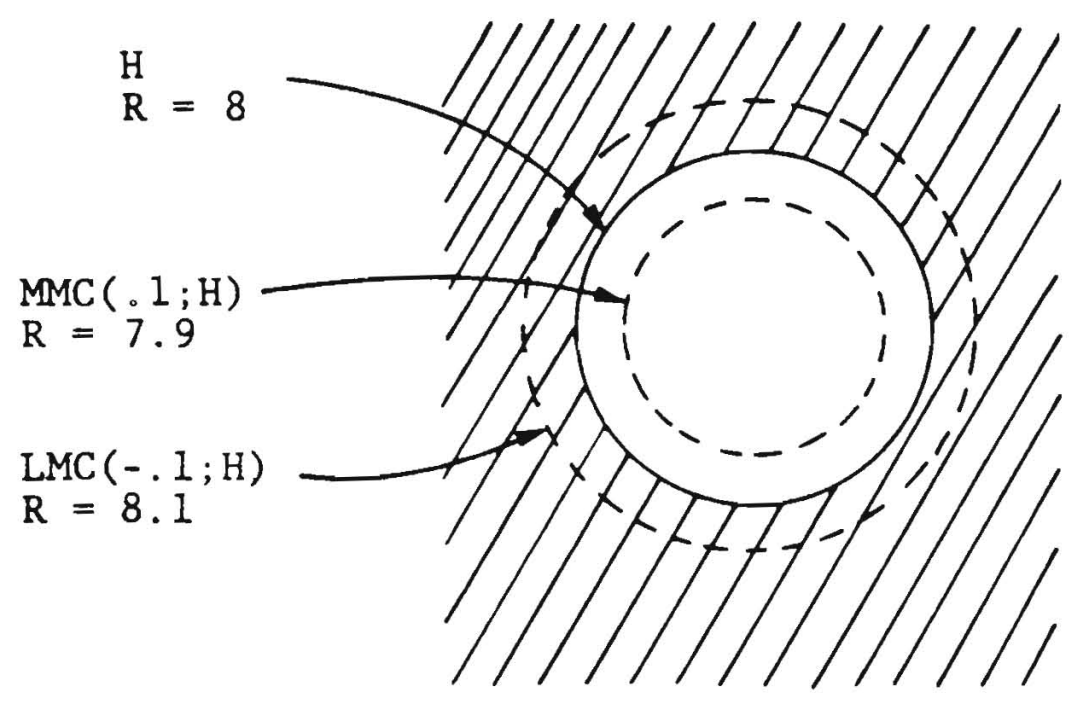

Figure 9-9

A cylindrical hole and its associated MMC and LMC solids.

More generally, a multiple-ofjset solid can be defined as follows. Consider $N$ nominal simple features $F_{i}$, with corresponding extended features $H_{i}$, and such 
that $F=\cup F_{i}$ is a composite feature, with a corresponding extended feature $H$. Denote by $E_{i}$ the homogeneously 2-D subset of $\partial H_{i}$ that also is a subset of $\partial H$. (Intuitively, $E_{i}$ is the contribution of $F_{i}$ to the boundary of $H$.) Associate with each $F_{i}$ a positive number $D_{i}$. The corresponding (positive) multiple-offset solid is

$$
O\left(D_{1}, D_{2}, \ldots ; H\right)=\bigcup_{i}\left\{p: p \in c H \text { and } \operatorname{Dist}\left(p, E_{i}\right) \leq D_{i}\right\} \cup H
$$

Intuitively, the multiple-offset solid is the result of adding to $H$ a layer of variable thickness; the thickness of the layer adjacent to feature $F_{i}$ is $D_{i}$. When all the $D_{i}$ are negative a (negative) multiple-offset solid is defined per

$$
O\left(D_{1}, D_{2}, \ldots ; H\right)=H-^{*} O\left(\left|D_{1}\right|,\left|D_{2}\right|, \ldots ; c^{*} H\right),
$$

which is a direct generalization of the earlier definition. Multiple-offset solids can be used to define multiple-offset MMC and LMC solids by considering vectors (arrays) of positive and negative offsets associated with features and interpreting $D_{p}$ and $D_{n}$ as vectors in the definitions of MMC and LMC given above. Figure 3-10a is a graphical multiple-offset specification for a simple solid, and Figures 3-10b and 3-10c show the corresponding MMC and LMC solids.

Armed with precise notions of MMC and LMC we can now define size tolerances. Let $G$ be an actual feature with corresponciing inominal feature $F$ and extended feature $H$. G satifies a size tolerance with single offsets $D_{p}, D_{n}$ if and only if there is a congruent instance $H^{\prime}=R(H)$, where $R$ is a rigid motion, such that

$$
G \subset \operatorname{MMC}\left(D_{p} ; H^{\prime}\right){ }^{*} \operatorname{LMC}\left(D_{n} ; H^{\prime}\right)
$$

In essence, $G$ must be within the "tolerance zone" that lies between an MMC and an LMC solid. The location and orientation of this tolerance zone are unspecified and can be selected so as to (hopefully) force $G$ to fit in it. When $D_{p}=-D_{n}=$ $T_{s} / 2$, one says simply "a size tolerance $T_{s}$ ".

When $D_{p}$ and $D_{n}$ are vector (multiple) offsets, $G$ satisfies the size tolerance if it lies in the composite tolerance zone delimited by the MMC and LMC solids, as in the single-offset definition above, and each individual simple $G_{i}$ satisfies the single size tolerance that corresponds to $F_{i}$. Figure 3-10d shows the composite tolerance zone that corresponds to the specification of Figure 3-10a, and also the individual tolerance zone that corresponds to the top face of the feature. 


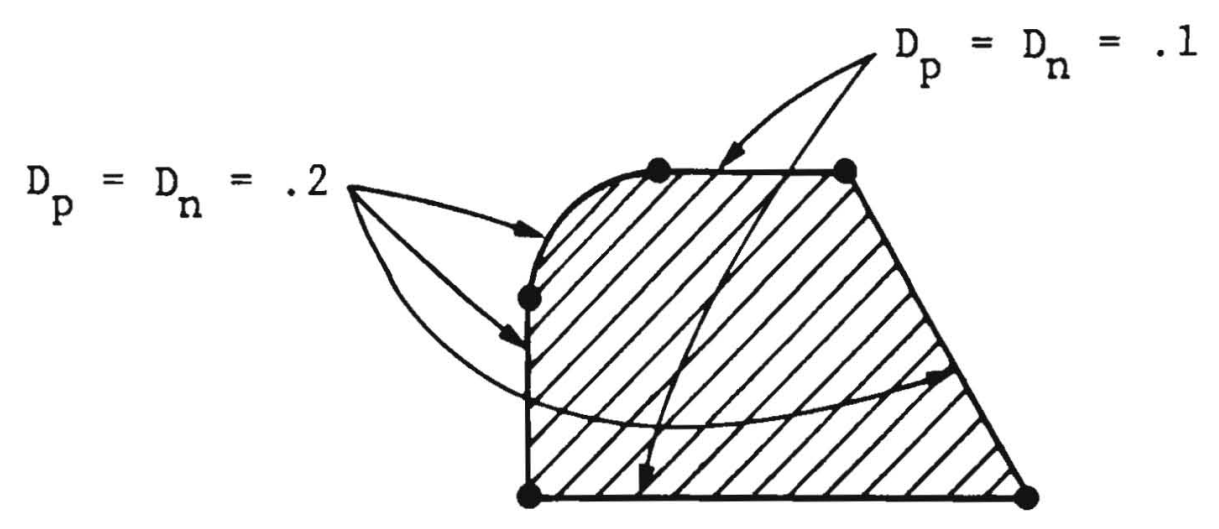

(a)

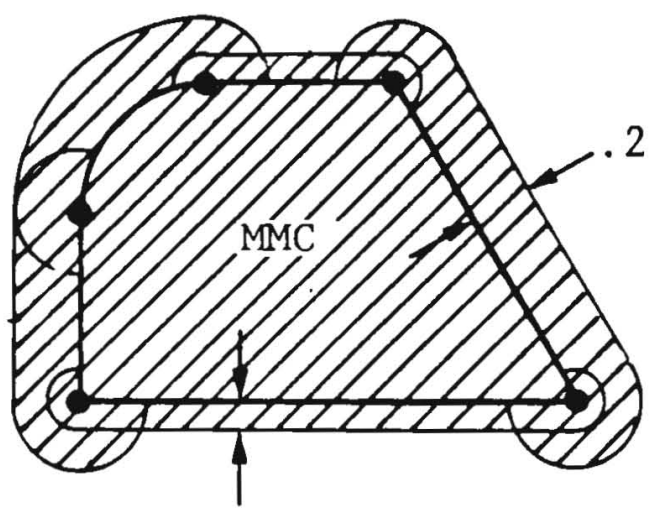

.1

(b)

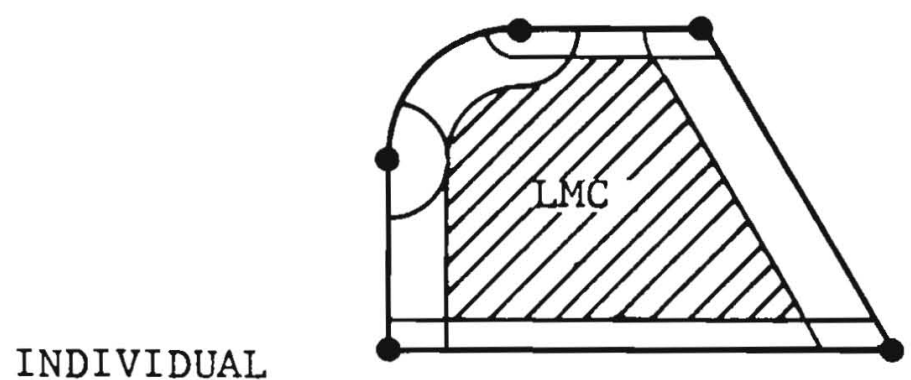

TOLERANCE ZONE

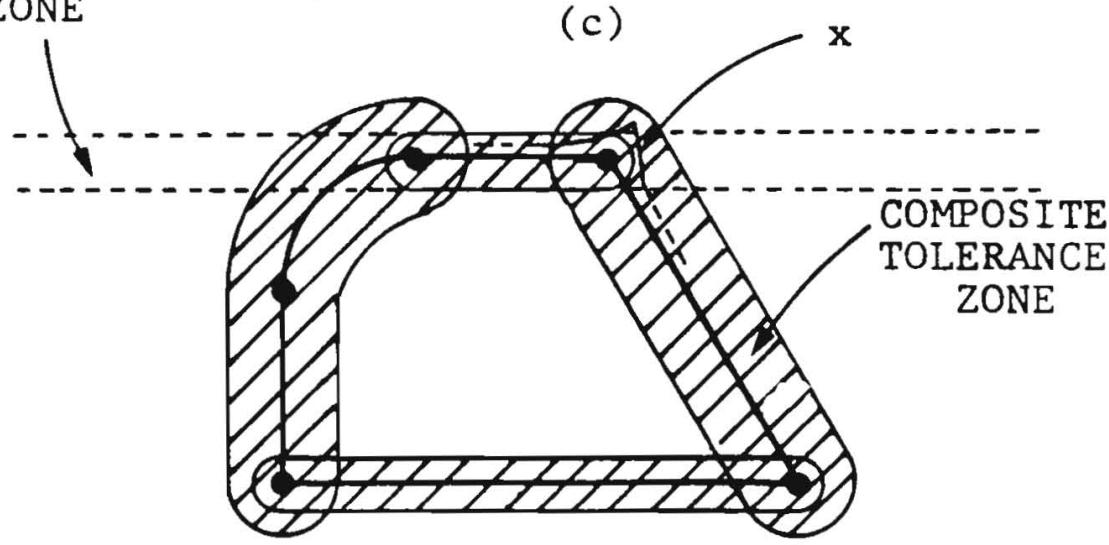

(d)

Figure 9-10

A multiple-offset size specification (a),

its associated MMC (b) and LMC (c) objects, and tolerance zone (d). 
The definitions above apply to any feature and therefore are extremely general. They largely agree with current practice for simple features such as cylindrical holes, but depart from current practice for more complex features, as in Figure 3-10d. Whether such departures are practically important remains to be seen. Note, however, that undesirable behavior such as that of surface $X$ in Figure 3-10d cannot occur, because $X$ cannot be segmented so as to satisfy simultaneously the individual size tolerances of the features and the slow variation constraint discussed in Section 3.2.

I chose to abandon in the definitions above the so-called "Taylor principle" of current practice because I was unable to generalize it to arbitrary features. My definition is somewhat more restrictive than current practices, which define lower size limits by means of a 2-D tolerance zone that can "float" within an MMC solid.

It is worth remarking that size tolerances, as defined above, are applicable also to planar features. For such features, size tolerance specification is essentiolly what is called "flatness" in current practice.

\subsection{Form, Orientation, and Runout Tolerances}

I propose to replace various special-case tolerances used in current practice (e.g., cylindricity, flatness) with a single form tolerance that applies to all features. In essence, an actual feature $G$ satisfies a surface form, tolerance specification with tolerance value $T_{f}$ if it lies in a tolerance zone of "width" $T_{f}$ and arbitrary "size" and orientation. A precise definition follows. Let $H$ be the extended feature that corresponds to $G$. Then, $G$ satisfies a form tolerance with value $T_{f}$ if there is a congruent instance $H^{\prime}$ of $H$ and two numbers $D_{1}$ and $D_{2}$ such that

$$
\begin{aligned}
& G \subset O\left(D_{1} ; H^{\prime}\right)-^{*} O\left(D_{2} ; H^{\prime}\right), \\
& D_{1}>D_{2}, \\
& D_{1}-D_{2} \leq T_{f} .
\end{aligned}
$$

For example, a cylindrical hole feature $G$ satisfies a form tolerance specification (called "cylindricity" in current practice) if $G$ lies in a cylindrical annulus defined by two coasial cylinders; the radii of the two cylinders are unspecified and need not equal the nominal, MMC or LMC radii, but they must differ by the specified form tolerance value.

Surface orientation tolerances are similar to surface form tolerances, but require datum specifications and imply tolerance zones correctly oriented with respect to the datums. Similarly, suriace (or total) runout tolerances require datum 
specifications and imply tolerance zones correctly located and oriented with respect to the datums. (Note that this definition of runout applies also to features that are not rotationally symmetric.)

The form and orientation tolerances described above apply to a surface as a whole. There are also similar tolerances - called curve tolerances in this paper that apply to curves lying on surfaces. Curve tolerances imply 2-D tolerance zones as explained below.

Consider an actual surface feature $G$, its corresponding nominal and extended features $F$ and $H$, and suppose that $G$ is constrained via a curve form tolerance with value $T_{c}$ with respect to a (nominal) plane normal to a vector $V$. Construct instances $F^{\prime}=R(F)$ and $V^{\prime}=R(V)$, where $R$ is a rigid motion, and the family of all planes $P_{i}$ normal to $V^{\prime}$. For each plane $P_{i}$ consider the intersection of $P_{i}$ with two oflset solids that "differ by $T_{c}$ " (as in the definition of surface form tolerances); this intersection defines a 2-D zone $Z_{i}$ "of width $T_{c}$ ". (Note that the various $Z_{i}$ need not be congruent.) Now apply a rigid motion $R_{i}$ in the plane $P_{i}$ to generate a tolerance zone $Z_{i}^{\prime}=R_{i}\left(Z_{i}\right) . G$ is "in spec" if and only if it is possible to perform the construction just described in such a way that the $Z_{i}^{\prime}$ zones include the intersections $G \cap P_{i}$, for all planes $P_{i}$. Figure 3-11 shows an example, which corresponds to what is called "roundness" in current practice: a cylindrical feature and an associated curve form tolerance with respect to the cylinder's axial direction. Observe in the figure that the 2-D annular tolerance zones $Z_{i}^{\prime}$ need neither be coaxial nor have the same radii; however, the two radii of each annulus must differ by the same amount $T_{c}$.

Curve form tolerances with respect to points and lines are defined similarly. They differ from the previous definition only in the method for constructing the planes $P_{i}$. For a curve tolerance with respect to a point, the $P_{i}$ are all the planes that contain the point, and for a tolerance with respect to a line, the $P_{i}$ are all the planes that contain the line.

The curve form tolerances just described are analogous to the form tolerances introduced at the beginning of this section, because the location and orientation of tolerance zones are not constrained by datum relationships. One could define curve tolerances with constrained orientation, but they don't seem to have a counterpart in current practices. If both the location and the orientation are fired with respect to a datum system one obtains generalizations of currently-used tolerances. For example, a curve tolerance with respect to a plane and with datum specification - called in this paper a curve runout tolerance with respect to a plane - is a generalization of a so-called "circular runout" tolerance. 


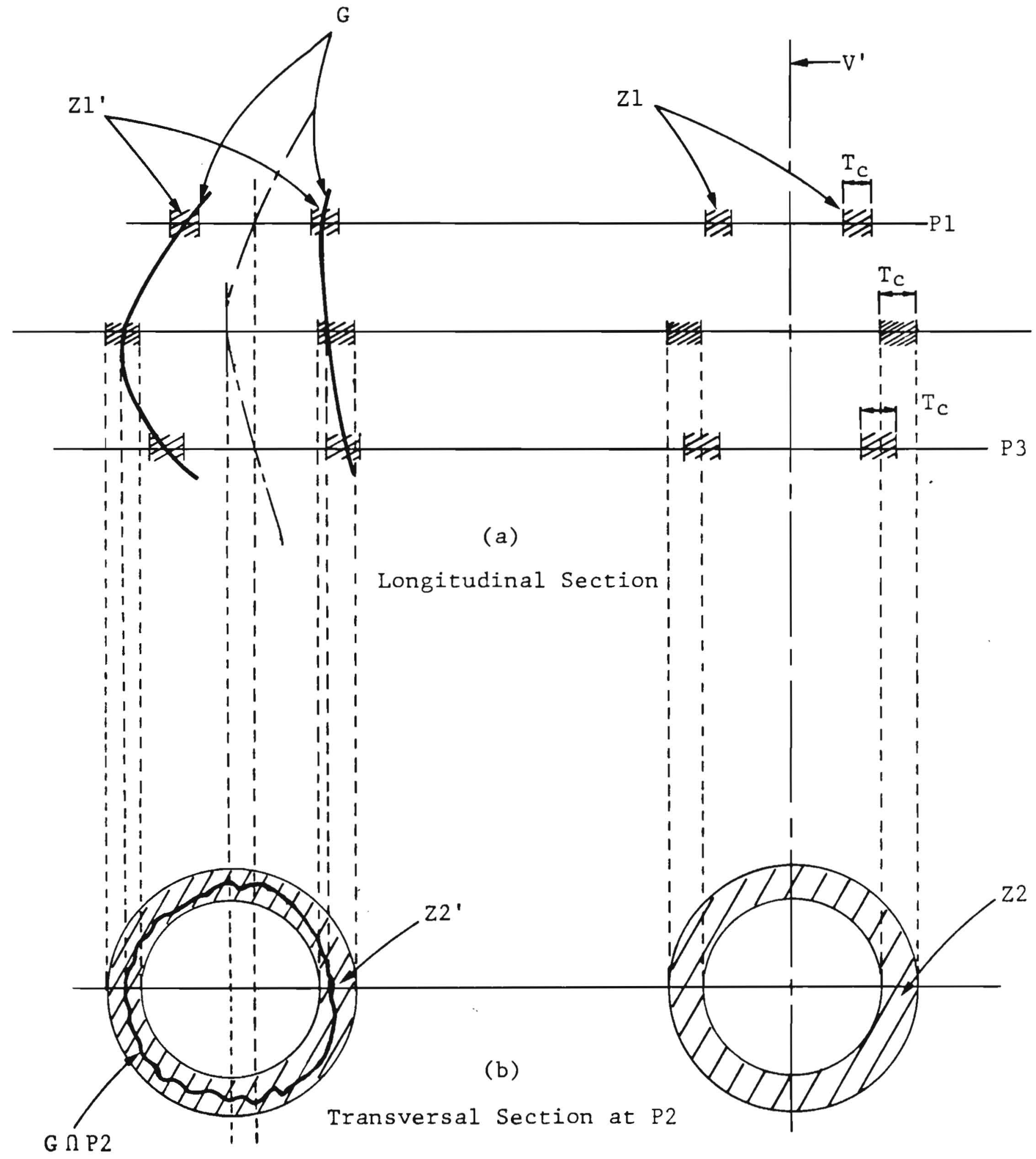

Figure 3-11

Curve tolerances for a cylindrical feature (roundness). 


\subsection{Position Tolerances}

An unqualified position tolerance with value $T_{p}>0$ and relative to a datum system defines a tolerance zone of width $T_{p}$ about a nominal feature. More precisely, an actual feature $G$ corresponding to a nominal feature $F$ and extended feature $H$ satisfies an unqualified position tolerance $T_{p}$ if

$$
G \subset O\left(T_{p} / 2 ; H^{\prime}\right)-{ }^{*} O\left(-T_{p} / 2 ; H^{\prime}\right),
$$

where $H^{\prime}$ is a congruent instance of $H$ that is positioned correctly, i.e., whose location and orientation relative to the datum system are precisely the same as those of $H$ relative to the nominal features that define the datum system. Observe that if $G$ satisfies a position tolerance $T_{p}$ then it also satisfies a size tolerance with positive and negative oifsets $T_{p} / 2$ and $-T_{p} / 2$, a form tolerance $T_{p}$, and an orientation tolerance $T_{p}$. Unqualified position tolerances are quite strict and do not reflect certain part mating relationships that are important in practice. The two types of qualified position tolerances described below also are necessary.

Given an actual feature $G$ corresponding to a nominal feature $F$ with size tolerances characterized by offsets $D_{p}$ and $D_{n}$, an $M M C$ position tolerance $T_{p}$ (with $T_{p} \geq 0$ ) relative to some datum system has the following meaning. First construct an instance $H^{\prime}$ of the extended feature $H$ at the appropriate location and orientation relative to the datum system. $G$ satifies its position constraints if $G \subset O\left(T_{p}+D_{p} ; H^{\prime}\right)$. When $D_{p}$ is a vector of offsets, $T_{p}$ should also be a vector, usually with all components equal to a single positive number.

RFS position tolerances have different semantics. They can be applied only to planar or symmetric features (i.e., features that can be associated with datums) and define constraints on the measured entities of the features (see Section 3.3).

Specifically, an RFS position tolerance is an assertion on a planar or symmetric feature that defines a solid tolerance zone within which a measured entify of the feature must lie. The tolerance specification consists of a tolerance value together with the "name" of the entity to which it applies and two "Bounding planes". Consider, for example, a feature symmetric about the $z$-axis. A tolerance specification consisting of (Axis $=z$, Value $=.1, Z_{\max }=3, Z_{\min }=0$ ) defines a cylindrical tolerance zone as shown in Figure 3-12.

An actual feature $G$ will satisfy this specification if and only if its measured axis $L$ (see Section 3.2) satisfies

$$
L \cap\left(P_{\max } \cap^{*} P_{\text {min }}\right) \subset \text { Tolerance Zone, }
$$

where $P_{\max }$ and $P_{\min }$ are the planar halfspaces whose intersection defines the "slab" of interest. 


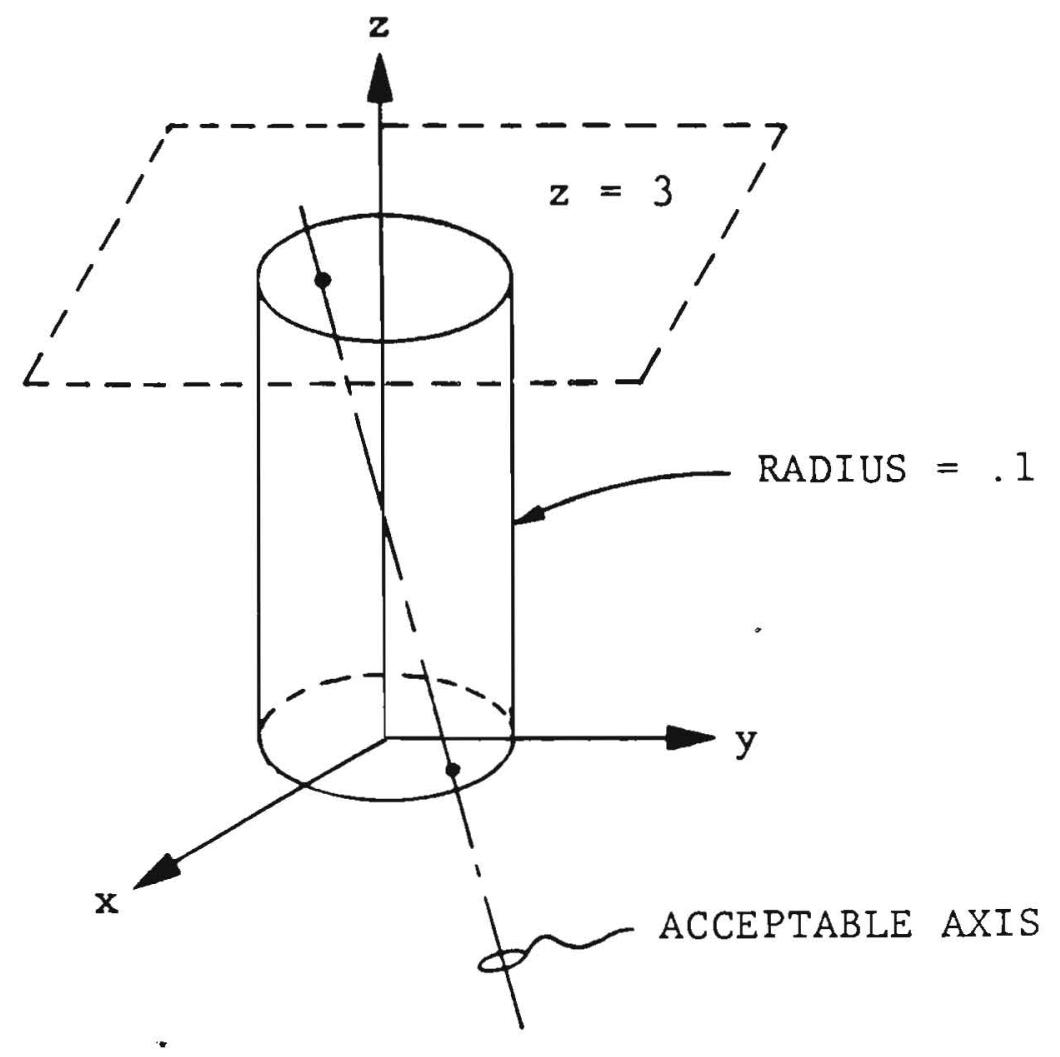

Figure 9-12

An RFS tolerance zone for an axis

RFS tolerances that apply to planes and centers are defined similarly, and correspond to cuboid and spherical tolerance zones, respectively. ${ }^{3-6}$ (Specifications for spherical tolerance zones do not include bounding-plane data.)

Current ANSI standards state that position tolerances are not applicable to planar features, but I see no reason for doing so.

3-6 I find the semantics of RFS position tolerances discussed above somewhat unpleasant because it involves bounded tolerance zones, instead of (generally) unbounded zones defined by offset solids. Unfortunately, I have not been able to provide a reasonable counterpart for current RFS practices via offset solids. 


\subsection{Validity of Tolerance Specifications}

A tolerance specification is valid if it defines an "acceptable" variational class. Unfortunately, a formal characterization of what constitutes an "acceptable" variational class is unknown, and therefore the validity of tolerance specifications is largely an open problem. ${ }^{3-7}$

The remainder of this section presents some informal thoughts on validity but does not attempt to settle the issues. Two validity conditions seem clear: the nominal representation must itself be valid and the union of all the nominal features must equal the nominal object's boundary. But what assertions must be required for validity? Can single assertions be unsatisfiable? Can collections of assertions have "contradictions" and therefore be unsatisfiable?

Observe that the theory prescribes tolerance zones that are always well defined, and are also "thick enough" to contain a nominal surface feature as well as an infinite family of features "close" to the nominal. This implies that single assertions are always satisfiable, provided that some relatively trivial conditions are met. (For example, daturn specifications must be valid, and symmetric-feature tolerances must not be applied to asymmetric features.) Observe also that it is impossible to construct contradictory assertions. Suppose, for example, that a feature has both a size tolerance $T_{6}$ and a form tolerance $T_{f}$. If $T_{f}>T_{s}$ then the form constraint is redundant (and can be ignored) because it is implied by the size tolerance; but if $T_{f}<T_{\varepsilon}$ then both tolerances apply independently. In either case there is no contradiction..$^{3-8}$

The conclusions are that an object cannot be overconstrained and (with minor qualifications, as noted above) any collection of assertions is satisfiable. But can an object be underconstrained? The answer clearly is yes. Conditions to ensure that constraints are "enough" are unknown. Intuitively, conditions should ensure that each feature lies in a tolerance zone of finite "thickness" and restricted position with respect to some datum system that is related to the object's master datum system. The important tolerances for specification validity appear to be size and position tolerances; others are optional but not needed for validity. My current conjecture is that a specification is valid if each feature's constraints imply a strict (unqualified) position tolerance with some finite, non-null tolerance value (see Section 3.7).

\section{...}

3-7 A conjecture: variational classes are regular closed sets in a hyperspace [NADL 78] whose elements are $r$-sets [REQU80].

3-8 When a nominal object is defined via geometric constraints (e.g., by requiring that certain distances or angles have given values) the situation is quite different, and it is easy to construct constraint sets that are unsatisfiable [REQU83]. 


\section{Conclusions}

Current tolerancing standards and practices must be tightened (formalized) considerably if we are to represent tolerancing information in computer-based geometric modelling systems in a form suitable for automatic tolerance analysis, automatic planning of manufacturing, assembly and inspection operations, and for other design and production activities.

The theory outlined in this paper is a step toward such formalization. The theory almost surely needs refinement, may require substantial modifications, and may even prove totally inadequate. However, it illustrates the level of precision required, and it shows that one can construct a reasonable theory of tolerancing that is based on a few general concepts rather than on an extensive set of specialcase considerations. The theory occasionally departs from current practices, but such departures may be unimportant.

The theory states that an object satisfies a tolerance specification if the object's bounding surfaces are within suitably defined regions of space called tolerance zones. Two types of tolerance zones are used:

1) Bounded tolerance zones are cylinders, parallelipipeds, or spheres within which axes, centerplanes, or centers of symmetric features must lie. These tolerance zones are used for RFS positioning.

2) General, usually unbounded tolerance zones are constructed via offset solids, and are used for all tolerancing except RFS positioning. General tolerance zones may include datum specifications. The distinctions between size, position and form-related tolerances lie in the specific rules for constructing the zones. (For example, is the location and orientation of a zone fixed, or can it "float"?)

Offset solids are defined in the theory in a specific and very general way, but much of the theory is applicable with any reasonable concept of offsetting. Parameterized CSG (Constructive Solid Geometry) or boundary representations [REQU80] presumably could provide alternative offsetting methods, but neither has been thoroughly investigated and both seem to raise delicate problems [REQU83].

Conventional $+/$ - tolerances are not supported directly; they must be replaced with functionally equivalent (or nearly so) constraints expressible in the theory. Usually, conventional tolerances can be replaced either by size tolerances or by position tolerances with explicit, rather than implied, datums.

Computational implementation of representation schemes based on the theory is relatively straightforward. In essence one need only provide in a GMS facilities for (1) defining and "naming" nominal surface features and associated entities (e.g. 
ases), (2) establishing relations between them, and (3) assigning attributes to them. (Offsetting semantics different from those described in Section 3.6 may pose quite different representational requirements.)

An experimental software system for representing tolerancing ("variationol") information is currently being implemented in the PADL-2 CSG-based geometric modeller at the University of Rochester [BROW82]. This subsystem is designed to support the tolerancing semantics described in this paper but provides "escape mechanisms" for user-defined semantics because it is unreasonable to expect a rapid change of tolerancing practices.

Two major questions must be addressed by future research:

- Does the theory satisfy adequately industrial requirements? (One should resist the temptation of "en riching" the theory to cater to special cases unless a critical analysis of industrial requirements shows that such cases are truly important.)

- Is the theory effective for applications such as tolerance analysis, and manufacturing and assembly planning, i.e., are such apnlications mathematically and computationally tractable in terms of the theory? 


\section{REFERENCES}

[ANSI73] "Dimensioning and Tolerancing", ANSI Standard Y14.5-1973, American Society of Mechanical Engineers, New York, 1973.

[BPOO81] R. A. Brooks, "Symbolic reasoning among 3-D models and 2-D images", Artificial Intelligence, vol. 17, nos. 1-3, pp. 285-348, Alugust 1981.

[BROO82] R. A. Brooks, "Symbolic error analysis and robot planning", International $J$. of Robotics Research, vol. 1, no. 4, pp. 29-68, Winter 1982.

[BFZOW82] C. M. Brown, "PADL-2: A technical summary", IEEE Computer Graphics and Applications, vol. 2, no. 2, pp. 69-84, March 1982.

[FITZ81] W. J. Fitzgerald, "Using axial dimensions to determine the proportions of line drawings in computer graphics", Computer Aided Design, vol. 13, no. 6, pp. 377-382, November 1981.

[HLL78a] P. C. Hillyard, "Dimensions and tolerances in shape design", Ph.D. Dissertation, Univ. of Cambridge, U.K., 1978.

[HILL78b] R. C. Hillyard and I. C. Braid, "Analysis of dimensions and tolerances in computer-aidled mechanical design", Computer Aided Design, vol. 10, no. 3, pp. 161-166, May 1978.

[HLL78c] R. C. Hillyard and I. C. Braid, "Characterizing non-ideal shapes in terms of dimensions and tolerances", ACM Computer Graphics, rol. 12, no. 3, pp. 234-238, August 1978.

[HOFF82] P. Hoffman, "Analysis of tolerance and process inaccuracies in discrete part manufacturing", Computer Aided Design, vol. 14, no. 2, pp. 83-88, March 1982.

[LEE82] Y. T. Lee and A. A. G. Requicha, "Algorithms for computing the volume and other integral properties of solids: II - a family of algorithms based on representation conversion and cellular approsimation", Comm. ACM, vol. 25, no. 9, pp. 642-650, September 1982 .

[LEVYT4] S. J. Levy, Applied Geometric Tolerancing. Beverly, MA: TAD Products Corp., 1974. 